\title{
Literatur, DaF und Kulturvermittlung im universitären Kontext in Ägypten
}

Ass.-Prof. Dr. Lamyaa Abdelmohsen Osman Ali Ziko

Abteilung für deutsche Sprache und Literatur

Philosophische Fakultät

Menoufia Universität 


\title{
Literature, German as a foreign language and transfer of culture in the university context in Egypt
}

\begin{abstract}
Questions such as what a clock has to do with a kitchen interrupt the treatment of German post-war literature on the example of Wolfgang Borchert's Kitchen clock (Die Küchenuhr) for a short while. The question draws attention to the fact that for some students a clock in the kitchen is not at all "self-evident", as they themselves perceived. Daily life and habits, which might be natural for German readers, are not always self-evident, daily, ordinary for Egyptian recipients. Especially the handling of literary texts presents the students with the inescapable confrontation of the self with the other.

The article looks back on 20 years of teaching experience in German studies, German literature and literary didactics and intends to summarize the following questions:

- What are the goals of literature lectures at the university?

- How can teaching of German language literature success didactically and interculturally?

- To what extent is it important or necessary to provide learners with intercultural skills through literary studies?

- What is the sustainability of literary education in lectures of German as a foreign language? What is the meaning of literature transfer with regard to job prospects or professional practice?
\end{abstract}

\section{Literatur, DaF und Kulturvermittlung im universitären Kontext in Ägypten}

\begin{abstract}
Fragen wie, was eine Uhr mit einer Küche zu tun habe, unterbrechen für eine kurze Weile die Behandlung der deutschen Nachkriegsliteratur am Beispiel Wolfgang Borcherts Küchenuhr. Die Frage macht darauf aufmerksam, dass für einige Studierende eine Uhr in der Küche gar nicht so „selbstverständlich“ ist, um beim Wortlaut der Kurzgeschichte Borcherts zu bleiben, wie es die Lehrkraft selbst wahrnimmt. Was für deutsche Leser als Selbstverständlichkeit, Alltag, Gewohnheit gilt, ist für ägyptische Rezipienten nicht immer selbstverständlich, alltäglich, gewöhnlich. Gerade das Behandeln von literarischen Texten stellt die Studierenden vor die unumgängliche Auseinandersetzung des Fremden mit dem Eigenen.

Aspekte der kulturellen Differenzen und interkulturelle Gesichtspunkte sind kein neues Thema in der Fremdsprachendidaktik. Interessant macht die Sache eigentlich nur, welche Erkenntnisse und Konsequenzen daraus für einen fremdsprachlichen Literaturunterricht gewonnen werden können; und auch die Erkenntnis, dass durch die neuen (besonders auch elektronischen) Medien die Kulturvermittlung eine neue Dimension gewinnt. Bei derartigen Fragen seitens der Studierenden heißt es für die Lehrkraft immer wieder, das Programm umzustrukturieren: Statt zunächst literaturwissenschaftliche Erkenntnisse zu erwerben, werden kulturelle, landeskundliche wie auch historische Informationen ermittelt und herausgearbeitet.

Der Beitrag blickt auf eine 20jährigen Unterrichtserfahrung in Germanistik, DaF und Literaturdidaktik zurück und beabsichtigt, sich resümierend mit folgenden Fragestellungen auseinanderzusetzen:
\end{abstract}


- Welche Ziele erstrebt der Literaturunterricht an der Universität?

- Wie kann die Vermittlung deutschsprachiger Literatur didaktisch und interkulturell gelingen?

- Inwieweit ist es wichtig oder notwendig, den Lernenden interkulturelle Kompetenzen in Bezug auf Literaturwissenschaft zu vermitteln?

- Welche Nachhaltigkeit hat die Literaturvermittlung im DaF-Unterricht? Bzw.: Welchen Sinn hat die Literaturvermittlung im Hinblick auf Berufsperspektive oder Berufsausübung?

Die Besonderheit der Auslandsgermanistik besteht nicht allein darin, dass die deutschsprachige Literatur mit anderen bzw. fremden Augen betrachtet und reflektiert wird. Ihre Erkenntnisse sind für die unterschiedlichen literaturwissenschaftlichen Diskurse nicht gering. Der wahre Gehalt der universitären Literaturvermittlung im Ausland liegt darin, dass sie nicht allein für Akademiker gedacht ist, sondern auch für die studierenden Rezipienten, die sich nach dem Studium nicht mehr damit beschäftigen werden. Es bleiben nur Bilder der Zielländer und Vorstellung über die Zielkulturen, die zum einen etwas Wahres enthalten, zum anderen ihrer Natur wegen „fiktiv“ sind.

\section{الأدب واللغة الألمانية كلغة أجنبية ونقل الثقافة فى الإطار الجامعى فى مصر}

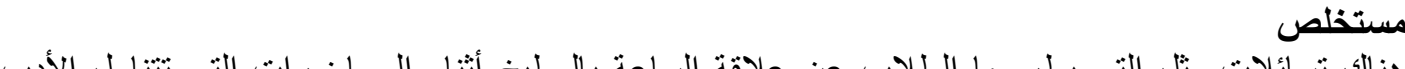

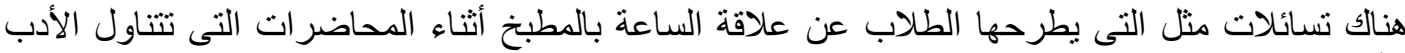

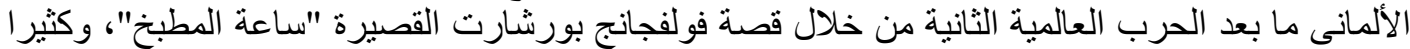

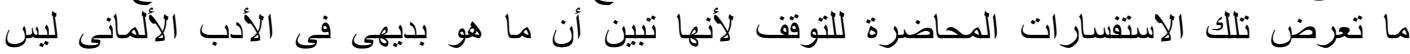

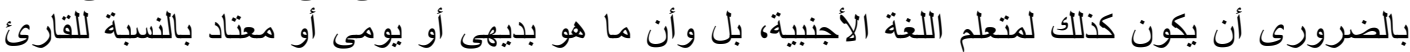

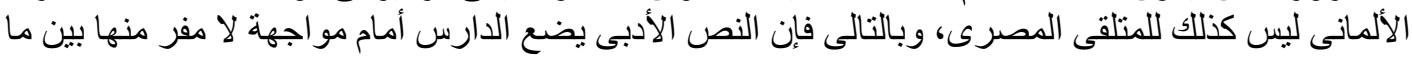
هو ذاتى وما هو غريب.

إن الاختلافات الحضارية والراتية الرؤى البينحضارية ليست موضو عا حديثا فى المناقثات التربوية الخاصة بتعلم

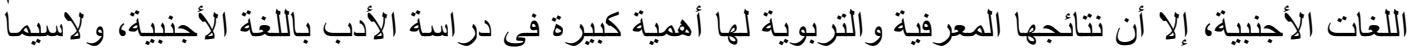

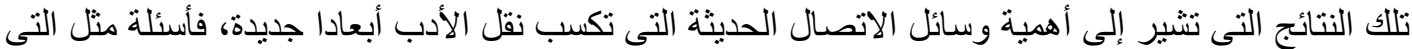

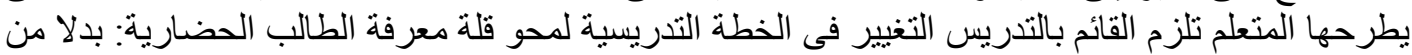

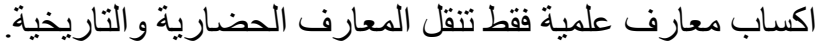
يعرض هذا البحث خبرة تدريس طويلة في مجال دراسة اللغة الألمانية وآدابها وتعليم الألمانية كلغة أجنبية

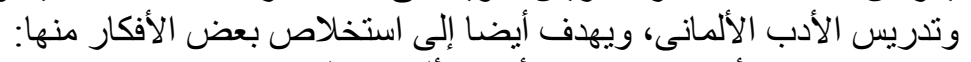

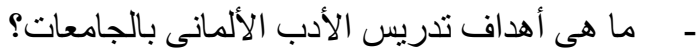

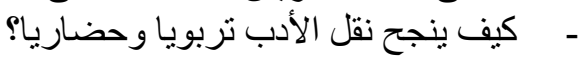

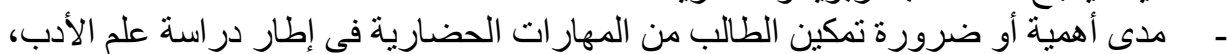

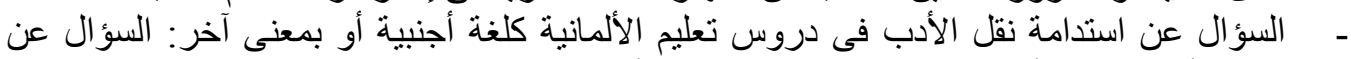

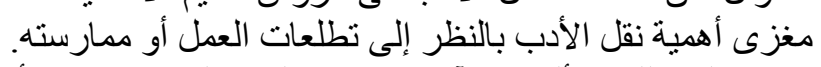
إن أهم ما بميز علوم اللغة الألمانية وآدابها خارج الهيلاد الإن الناطقة بها هو أنها ثُرى وتُعكس بعين الآخر ، ويتبلور

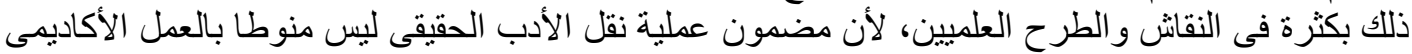

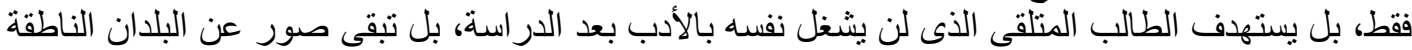

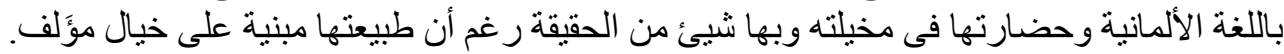




\section{Literatur, DaF und Kulturvermittlung im universitären Kontext in Ägypten ${ }^{1}$}

\section{Umriss der Thematik}

Das Studium der deutschen Sprache und Literatur gehört zu den etabliersten fremdsprachigen Sprachstudienangeboten in Ägypten, die besonders in den letzten Jahren einen regelrechten Umschwung erfahren haben. Der Grund liegt offensichtlich darin, dass der Bedarf an deutschkundigen Arbeitnehmern besonders in Firmen, die telefonischen Kundendienst auf Deutsch anbieten, rasant gestiegen ist. Da die meisten universitären Einrichtungen dieses Studienangebot vor allem jenen ermöglichen, die nur geringe bzw. keine Deutschkenntnisse haben, konzentrieren sich die ersten 2-3 Semester (von insgesamt acht) auf die Vermittlung der deutschen Sprache in den vier Fertigkeiten Lesen, Schreiben, Hören und Sprechen wie auch Übersetzung. Eine sprach- bzw. literaturwissenschaftliche Ausbildung erfolgt in der Regel ab der Mitte des Studiums und später optional in den postgradualen Studien. Kulturelle Inhalte über die deutschsprachigen Länder werden in der Regel mittels gängiger Lehrwerke, aber auch durch die Übersetzung Arabisch/Deutsch und nicht zuletzt durch gezielte landeskundliche Lektüre vermittelt (dazu Ali 2009; Ziko 2012).

Es sind gerade jene kulturbezogenen Fragen auffällig, die während des fremdsprachlichen Literaturunterrichts und der literaturwissenschaftlichen Seminare gestellt werden, wie z. B. „Was hat eine Uhr mit einer Küche zu tun?“ gestellt von einer Studentin im achten Semester während der Behandlung von Wolfgang Borcherts Kurzgeschichte Die Küchenuhr im Rahmen eines Seminars zur deutschsprachigen Gegenwartsliteratur. Der fremdsprachliche Literaturunterricht außerhalb der deutschsprachigen Region hat durch die Fremdperspektive der Studierenden eine andere Dimension erfahren, die mit der Wende zum 21. Jahrhundert auf Resonanz in der didaktischen DaF-Diskussion gestoßen und mittels inter- bzw. transkultureller Perspektiven zu neuen Einsichten, Erkenntnissen und Ansätzen gekommen ist.

Das Arbeitsgebiet des fremdsprachlichen Literaturunterrichts mit Deutsch hat sich seit Jahrzehnten nicht nur methodisch-theoretisch entwickelt, sondern auch viele praktische Anwendungen hinter sich, die Eingang in die literaturdidaktischen Nachschlagewerke gefunden haben. Ausgehend vom Konzept des Fremden und Eigenen bei Wierlacher (1985) sind besonders die Konzepte der Inter- und Transkulturalität, des Cultural Turns u.ä. entstanden und haben neue Gruppierungen und Tendenzen mit sich gebracht, die Kultur, Kulturverständnis und Kulturvermittlung mittels Literatur im Rahmen einer kulturwissenschaftlichen Diskussion einbeziehen. Es stellen sich hierbei Fragen nach $\mathrm{zu}$ vermittelnden

\footnotetext{
${ }^{1}$ Der Beitrag beruht auf einem Vortrag mit dem Titel „'Was hat eine Uhr mit einer Küche zu tun? Literatur, DaF und Kulturvermittlung in Ägypten. Eine Bilanz.“, der anlässlich der vom DAAD organisierten Konferenz ,alMena - Germanistik und DaF in der Menaregion“ vom 14.-16.5.2017 in Kairo gehalten wurde.
} 
literarischen Texten, nach Angehensweisen und Behandlungsmöglichkeiten, aber auch nach der vielseitigen Einsetzbarkeit im fremdsprachlichen Unterricht schlechthin (dazu Altmayer 2002; Hofmann 2006; Fäcke/Wangerin 2007; Esselborn 2010; Altmayer/Dobstadt/Riedner/Schier 2014; Altmayer/Dobstadt/Riedner 2014; Kramsch 2011 und 2016 u.v.m.). Die ägyptischen Germanisten bemühen sich neben Bestandaufnahmen, die die Entstehung, Entwicklung und Herausforderungen der germanistischen Studien in Ägpyten veranschaulichen (Maher 2007; Khattab 2010), auch um eine Auseinandersetzung mit dem Fach Germanistik im interkulturellen Kontext. Ein Zeugnis dafür ist der Sammelband Interkulturelle Kommunikation (Czucka/Neuland 2011), worin in Bezug auf Literatur unter anderem Massoud (2011) sich mit der Rezeption der ägyptischen Germanistik im deutschsprachigen Germanistikraum, also mit der Wechselwirkung der germanistischen Forschung, auseinandersetzt. Es werden ferner Themen angeschnitten, insbesondere inbezug auf die Wahrnehmung der islamisch-arabischen Welt in der deutschsprachigen Literatur (Helal 2011), wie auch komparatistische, rezeptionsorientierte Untersuchungen oder postkolonialistische Ansätze (El Dib 2011; Salama 2011) vorgestellt. Diese Diskussionen beabsichtigen vorrangig, die Implementierung eines Masterstudiengangs für Interkulturelle Kommunikation mit anwendungsorientierten Zielsetzungen in Ägypten zu rechtfertigen. Konkrete literarische Analysen und Interpretationen veranschaulichen demzufolge die abzudeckenden Themenbereiche und Forschungsschwerpunkte für ein derartiges postgraduales Projekt. Welche methodisch-theoretischen Grundlagen und didaktischen Vorarbeiten, die im Grundstudium erzielt werden könnten, dazu vonnöten sind, werden nicht angesprochen und sollen im vorliegenden Beitrag thematisiert werden.

Innerhalb des interkulturellen Kontextes und Diskurses führen besonders die vielfältigen Einsatzmöglichkeiten von Literatur vermehrt zur Erörterung der Bildungsfunktion von Literatur. Dabei stellt Esselborn (2010) fest:

Offener denn je sind die Fragen, was literarische Texte überhaupt zu leisten vermögen, welche soziale Funktion, welcher Bildungswert ihnen zukommt. (Esselborn 2010: 18)

Diese Fragen stellen sich die Literaturdidaktiker gleichwohl für den Mutter- wie auch Fremdsprachenunterricht, besonders im Rahmen der Bildung von interkultureller Komptenz oder der Förderung interkultureller Kommunikation. Auf die Frage nach dem „Bildungssinn literarischer Texte“, wie Lothar Bredella (2007) seinen Beitrag untertitelt, gibt er folgende Antwort:

Sie [Literarische Texte] wollen uns nicht von der Welt befreien, sondern sie uns aus einer neuen Perspektive zeigen und dabei auf unsere Wertvorstellungen und Sichtweisen einwirken. Wir lassen somit in der Rezeption eine fremde Welt entstehen, die uns bestimmt. (Bredella 2007: 41) 
Der Begriff der Rezeption wie auch der Rezeptionsästhetik hat sich in den letzten Jahren in der literaturdidaktischen Diskussion besonders im Rahmen des fremdsprachlichen Literaturunterrichts festgesetzt. Zahlreiche Beiträge gehen dabei auf Namen wie Jauß oder Iser ein, beschäftigen sich mit formalistischen oder konstruktivistischen Leitideen, denen sie zustimmen oder die sie ablehnen. In weiteren Beiträgen dienen derartige Auseindersetzungen schon seit den achtziger Jahren zu der interkulturellen Diskussion (dazu bspw. Ehlers 2010; Riedner 2010, Ewert 2010).

Der vorliegende Beitrag hat vor diesem Hintergrund nicht die Absicht, sich auf diese oder jene veröffentlichten Ergebnisse ausführlich einzulassen. Es ist auch keine Zusammenstellung von literaturdidaktischen Lösungen zu Problematik und Sinn der Behandlung von Literatur in der ägyptischen Germanistik angestrebt. Vielmehr sollen Reflexionen, die einige Erkenntnisse aus jahrelanger Erfahrung mit fremdsprachlichem Literaturunterricht an ägyptischen Universitäten zusammenführen, zum Ausdruck gebracht werden, die für die weitere Diskussion um den Umgang mit Literatur und Literaturwissenschaft im universitären Rahmen und für das Fach DaF von Belang sein können. Jene theoretischen und praktischorientierten Beiträge, Einführungen und Sammelbände bestätigen in diesem Zusammenhang eine relevante didaktische Entscheidung: Dass der Umgang besonders der produktive - mit Literatur auch im fremdsprachigen Germanistikstudium außerhalb der deutschsprachigen Region viele Ziele verwirklichen kann. Einige Aspekte, die z. B. Esselborn (2010), Choi (2002), Tütken (2006), Augart (2015) u.v.a. aus eigenen Praxis-Erfahrungen in ihren Beiträgen oder Nachschlagewerken vorlegen, werden auch in diesem Beitrag wiederzufinden sein, was eigentlich nur belegt, dass sich die Beschäftigung mit fremdsprachlicher Literatur im Studium mit denselben Gedanken auseinandersetzt, die man im praktischen Unterricht entwickelt. So lenkt Esselborn seine Fragestellung im Hinblick auf die Vermittlung von deutschsprachiger Literatur darauf hin, dass bei der
[...] Vermittlung deutscher als fremder Sprache und Kultur zu klären [wäre], was denn die leitende Idee bei der Beschäftigung mit fremdsprachlicher und fremdkultureller Literatur sein könnte, wieweit insbesondere fremdsprachige Literatur fremde Kultur repräsentieren und zugänglich machen kann. Ferner ist zu fragen, inwiefern sich eigen- und fremdkulturelle literarische Lektüre unterscheiden, welche institutionellen Lernziele damit vereinbar sind, welche didaktischen Auswahlkritierien für Leselisten bzw. für einen speziellen literarischen Kanon sich daraus ergeben und wie schließlich eine methodisch- didaktische Umsetzung aussehen könnte usw. (Esselborn 2010: 18f)

Die Lehrkraft als Vermittler von fremdsprachiger Literatur muss sich demnach bei der Behandlung von literarischen Texten mit Aspekten des Kontextes, der Repräsentativität und Zugänglichkeit zur deutschsprachigen Literatur beschäftigen, die Lese-Gewohnheiten der Fremdsprachenlerner berücksichtigen, wie auch die 
Ziele der Institution, die nicht zuletzt die Auswahl des zu behandelnden literarischen Textes steuert und akademische Zielsetzungen stellt, was folglich die Methodik und Didaktik des Vermittlungsprozesses beeinflusst. Bei alldem hat die Lehrkraft auch immer das Ziel vor den Augen, das Interesse des Lerndenden für die fremdsprachige Literatur zu wecken.

Das sind durchaus komplexe Bedingungen, die sich dem fremdsprachlichen Literaturunterricht zusammenfügen, und die für das universitäre Deutsch-Studium eine Herausforderung stellen; denn die akademische Ausbildung eines ,Germanisten' im Ausland verlangt zudem die Vermittlung von wissenschaftlichen Erkenntnissen, Kompetenzen und Fähigkeiten in der Fremdsprache. Gleichzeitig sei hier vorweggenommen, richtet sich die Entscheidung der Studierenden für dieses Studienfach nicht einzig an einer, germanistischen" Ausbildung. Die deutsche Sprache sehen sie weitesgehend eher als Möglichkeit, sich im Arbeitsmarkt einen Platz zu sichern oder auch als Eintrittskarte zu deutschen Universitäten. Das bedeutet wiederum für die Lehrkraft, den Studierenden weitere Kompetenzen als nur wissenschaftliche zu vermitteln.

Die Studiensituation an ägyptischen Abteilungen, die eine germanistische Ausbildung anstreben, steht vor diesem Hintergrund vor vielen Hürden. Die erste liegt allein darin, dass die Mehrzahl der ägyptischen Studierenden ihr Studium der deutschen Sprache und Literatur mit geringen Grundkenntnissen über die deutsche Sprache, die sie in der Oberschule erworben haben, antreten. Diese sprachliche Schulausbildung erlaubt es bei weitem nicht, die angehenden Studierenden in einen fortgeschrittenen Lehrinhalt einzuführen. Das Studium beginnt daher mit einem Niveau von A1, worin es erst darum geht, den Studierenden erneut die Grundlagen der deutschen Sprache zu vermitteln (dazu Ali 2009; Ziko 2012). Die Studierenden fangen sprachlich demzufolge fast bei Null an und merken bald, dass vieles in der Schule anders vermittelt wurde; aber vor allem: dass sie nicht einfach nur die Sprache Deutsch lernen. Sie werden mit der Tatsache konfrontiert, dass die Universität kein Sprachinstitut und das Studium kein Sprachkurs ist. Erwartungen und Realität geraten hier aneinander; denn neben der Vermittlung von deutschsprachiger Kultur, Gesellschaft, Gewohnheiten und Lebensweisen usw. setzen sich die Abteilungen an den ägyptischen Universitäten ferner zum Ziel, ägyptischen Studierenden Fachwissen über Sprache und Literatur zu vermitteln. Diese Inhalte sind einerseits erforderlich für eine akademische Ausbildung und andererseits sollen sie die Studierenden befähigen, sich in der Berufswelt durchzusetzen.

Der Umgang mit literarischen Texten ist demzufolge nicht per se als fremdsprachiger Literaturunterricht $\mathrm{zu}$ betrachten, sondern muss insbesondere für die Germanistik (als DaF, Internationale Germanistik oder fremdsprachige Philologie) eine neue Einteilung erfahren, die für die germanistische Ausbildung, aber auch für den Spracherwerb und damit für die theoretischen Überlegungen, die 
methodisch-didaktische Umsetzung und die Qualität des Angebots entscheidend sein können.

\section{Theoretische Rahmenbedingungen des universitären fremdsprachlichen Literaturunterrichts}

In der literaturwissenschaftlichen Diskussion zur Vermittlung von Literatur und literaturwissenschaftlichem Wissen innerhalb eines systematischen Studiums der deutschen Sprache und Literatur im Ausland wird nicht unberechtigt eine Systematisierung oder Didaktisierung von Literatur und Literaturwissenschaft innerhalb des Faches Deutsch als Fremdsprache bemängelt, dessen Strukturalisierung ebenfalls beklagt und/oder umstritten diskutiert wird (s. Altmayer 1997; Esselborn 2010). Zu sehr und zu lange war man in der Diskussion damit beschäftigt, nach der Auswahl geeigneter literarischer Texte, nach zu vermittelnden Inhalten wie Mentalitätsgeschichte, Landeskunde, Kultur oder gar Ästhetik zu fragen. Bezogen haben sich diese didaktischen Auseinandersetzungen meist auf Deutschlernende und Studierende im deutschsprachigen bzw. europäischen Raum; vermehrt in den letzten Jahren auch auf jene, die sich außerhalb dieser (sprach-geographischen) Räume befinden. Grundsätzlich sind laut Esselborn bei der Vermittlung des Deutschen als Fremdsprache verschiedene Vermittlungsbereiche zu unterscheiden:

[...] vom (unterschiedlich motivierten) Fremdsprachenunterricht in speziellen Institutionen über das (pädagogisch fundierte) Fach Deutsch an Schulen im Ausland (bzw. Deutsch für Migrantenkinder in Deutschland) bis zum Germanistikstudium für Nicht-Muttersprachler an Universitäten im In- und Ausland bis zur Privatlektüre und ihrer Unterstützung etwa durch Rezensionen, Anthologien und Textkommentare. (Esselborn 2010: 29)

Relevant ist diese Unterscheidung zwischen den Zielgruppen und den Vermittlungsbereichen in dem Sinne, dass sie Konsequenzen auf Ziel und Absicht der Literaturvermittlung, Textauswahl und schließlich auf die erworbenen Erkenntnisse haben. Um diese Ambitionen zu erreichen, sind jedoch zwei Blockaden zu überwinden: die sprachliche und literaturkonzeptionelle.

In der universitären Praxis ist zunächst die Sprache jene Blockade, die den Empfang von und die Auseinandersetzung mit Literatur und Literaturwissenschaft erschwert. Zudem sind die Studierenden noch $\mathrm{zu}$ sehr auf die frontale Vermittlungsmethode von wissenschaftlichen Inhalten orientiert, bei der sie Inhalte aufzunehmen haben, um sie in Klausuren wiedergeben zu können. Der Sinn und das Ziel eines Fremdsprachenstudiums ragen jedoch über diesen evaluierenden Aspekt hinaus, denn sie sollen ferner kommunikative und intellektuelle Fähigkeiten erwerben und entwickeln. Steht man vor der blockierenden Haltung der Studierenden, mit fremdsprachiger Literatur umzugehen, schlussfolgert man meist 
auch deren Desinteresse an Literatur schlechthin. Man stellt als Lehrkraft oft auch infrage, wie und ob sie die muttersprachliche Literatur rezipieren und welches Hintergrundwissen sie aus der schulischen Ausbildung mitgenommen haben. Augart (2015) stellt hierbei fest:

Jedoch kann man sagen, dass im Literaturunterricht in der Schule die literarische Wirklichkeit oft weit von der Lebenswelt des Lesers entfernt ist, dass der Unterricht eher auf Wissenserwerb und Wissensrekapitulation denn auf einen kreativ-produktiven Umgang ausgelegt ist. (Augart 2015: 578)

Das trifft größtenteils ebenfalls auf die Rezeption und Vermittlung von Literatur und literarischen Werken an ägyptischen Schulen und auch Universitäten zu. Allerdings kann man nicht pauschal davon ausgehen, dass die jungen StudentInnen oder gar auch SchülerInnen kein Interesse am Lesen haben und dass digitale Medien das Interesse oder gar die Lust daran verdrängt haben. Man wird eines besseren belehrt: Besonders die ägyptische Gegenwartsliteratur ist bei vielen jungen Menschen in den Schulen und Universitäten populär. Der Dozent ist allerdings immer noch auf die „Klassiker“ der arabischen Literatur fixiert. Auch wenn die StudentInnen zunächst - wie auch im Fall bei Augart - keine Antwort auf Leseerfahrung geben, heißt das noch lange nicht, dass sie literarische Lektüre meiden. Die Hemmung seitens der Studierenden liegt vor allem darin, den Dozenten nicht mit ihrem Geschmack zu enttäuschen. Es muss ihnen das Gegenteil bewiesen werden, indem man sie mit eigenen Kenntnissen und Leseerfahrungen mit der zeitgenössischen Literatur konfrontiert. Das Ergebnis: Sie liefern weitere (dem Dozent unbekannte) Beispiele zur ägyptischen Literatur und bestätigen somit, dass Literatur zum Bestandteil ihrer Freizeitbeschäftigung gehört. Und: Die Rolle der Literatur unter der jungen Generation hat ihre unterhaltende und vor allem ästhetische Funktion nicht verloren. Von daher ist es auch gewagt zu behaupten, dass es in manchen kulturellen Regionen noch an deutlichen Literaturkonzepten mangele, wie Esselborn annimmt und fordert:

Eine systematische Bestandsaufnahme der Rolle von Literatur in den unterschiedlichen nationalen Bildungskonzepten - vor allem im Vergleich mit den idealistischen deutschen Traditionen - ist noch ein dringendes Desiderat. Untersuchungen zu den außereuropäischen (speziell den älteren und den oralen) literarischen Kulturen zeigen, dass dort entweder noch gar kein Konzept von ,Literatur' vorhanden ist, wie es in Europa im 18. Jahrhundert entwickelt wurde, oder dass die erst später erscheinenden Konzepte wie das chinesische „wen“ (seit dem 3. Jahrhundert), das „kāvya“" im Sanskrit oder das arabische „adab“ lediglich ein säkulares Schreiben (aus verschiedenen Lebensbereichen) mit kulturellem Prestige und von allgemein erzieherischem Wert, als Teil des für gebildete Personen nötige intellektuelle Gepäcks, umfassten. Trotz europäischer Einflüsse hat z.B. die Idee einer 
ästhetischen Autonomie keine universale Akzeptanz gefunden. (Esselborn 2010: 31)

Diese Behauptung ist pauschalisierend und unzureichend belegt. Unwissen oder mangelnde Informationen über die Literaturkonzepte anderer Kultur heißt nicht, dass sie nicht existieren. Bei genauer Betrachtung gelten die Kriterien Prestige, Erziehung und Intellektualität allein nicht mehr für die Leser außereuropäischer Regionen, sondern seit langem und vor allem auch das Kriterium der ,ästhetischen Autonomie".

Dieser kurze Exkurs beabsichtigt einen Beweis dafür zu liefern, dass die gegenwärtige ägyptisch-arabische Literatur den jungen StudentInnen geläufig ist. Als Zugang zur deutschen Literatur ist die Kenntnis der eigenen arabischen Literatur notwendig. Diese Erkenntnis ist sowohl für die akademische Lehrkraft als auch für die Studierenden eine gute Basis für die Vermittlung fremdsprachiger Literatur und literaturwissenschaftlicher Inhalte.

\section{Didaktische Vorarbeit für den fremdsprachlichen Literaturunterricht}

Die vorangegangenen Gedanken und Beschreibungen beantworten allerdings noch nicht die Fragestellung nach den Zielen des fremdsprachlichen Literaturunterrichts an der Universität. Neben den sprachlichen Zielen wie der Förderung des Leseverstehens und der sprachlichen Sensibilisierung stehen noch weitere Aspekte im Mittelpunkt. Hierzu zählen zum einen die Vermittlung landeskundlicher Informationen: Familienbeziehung, zeitgebundene Themen, Konflikte innerhalb der Gesellschaft, politische Landschaft usw. Ferner dienen die verschiedenen literaturwissenschaftlichen Fächer einerseits der Vermittlung von Literaturgeschichte, Gattungen und literarischen Themen und andererseits der Aneignung von wissenschaftlichen und berufsbezogenen Kompetenzen. Um diesen Zweck zu erfüllen, werden unterschiedliche Fächer aus dem Bereich der Literaturwissenschaft angeboten: Seminare zu den unterschiedlichen Epochen der deutschen Literaturgeschichte, zu den unterschiedlichen Gattungen, Einführungen in die Literaturwissenschaft, Komparatistik. Sie beabsichtigen, das wissenschaftliche Arbeiten mit literarischen Texten, in denen die Studierenden mit dem Begriffsapparat und den Analyseverfahren vertraut gemacht werden: z. B. was einen literarischen Text ausmacht, wie sich literarische Gattungen von einander unterscheiden, wie eine Figur beschrieben und charakterisiert werden kann, wie Zeit und Raum in einem literarischen Text konzipiert sind, wie lyrische Texte verstanden und interpretiert werden, wie der Aufbau dramatischer Texte analysiert und wie sich ein Thema in einem Prosawerk entfaltet u.v.m.

Diese Zielsetzung bedingen allerdings Vorarbeiten, um ein Verständnis für die deutschsprachige Literatur auf sprachlicher und (inter)kultureller Ebene zu realisieren. Dieses Verständnis ist für eine literaturwissenschaftliche Auseinandersetzung mit literarischen Texten relevant. Jost Schneider (1998) fordert 
die Erfüllung von drei Notwendigkeiten insbesondere bei der Figurenanalyse, die für den fremdsprachlichen Lernenden für die Arbeit mit der fremdsprachlichen Literatur überhaupt erforderlich sind:

Erstens bedarf es zur kompetenten Figurenanalyse umfänglicher sozialhistorischer Kenntnisse, damit die Stellung einer Figur im sozialen Raum überhaupt erkannt und sachangemessen beschrieben werden kann. [...] Zweitens muß der Textinterpret [...] eine außergewöhnliche Fähigkeit zur Selbstdistanzierung und Selbstrelativierung aufbringen, um auch diejenigen Milieus sachlich beschreiben zu können, die ihm selbst fremd oder gar verhaßt sind. [...] Drittens bedarf es einer kontrollierten Kreativität im Hinblick auf erforderliche Analogiebildungen, die ein Verstehen der Struktur von besonders phantastischen sozialen Räumen in sehr alltags- und gegenwartsfernen Texten ermöglichen. (Schneider 1998: 39f)

Die wissenschaftliche Beschäfigung mit Literatur ist folglich gezielt in der Lage, die Zielkultur $\mathrm{zu}$ vermitteln, ein Verständnis für diese $\mathrm{zu}$ entwickeln und interkulturelle Kompetenzen zu fördern.

Die systematische Vermittlung literaturwissenschaftlicher Inhalte setzt demzufolge in der fortgeschrittenen Phase des Studiums an, nachdem eine sprachliche Grundlage aufgebaut ist, die den Studierenden erlaubt, literarische Texte zu verstehen und mit ihnen umzugehen. Gleichzeitig kommen verschiedenen Fächern zur Landeskunde eine wichtige Rolle zu: Sie informieren über im Zielland und in der Zielkultur übliche und gewohnte Gegebenheiten. Damit wäre der sprach- und landeskundedidaktische Teil geklärt. Der interkulturelle Teil erweist sich als eine weitere Hürde, denn damit verbunden ist vor allem die Kenntnis des Lehrenden über Zielland und Zielkultur. Um diese Problematik zu veranschaulichen, seien einige Situationen aus den ersten Semestern vorzuführen, in denen die Annäherung zur fremden Kultur den Studierenden neue Einsichten brachten.

- Eulen gelten in Ägypten als Unglücksbringer; in Deutschland bringen sie Glück. Die entscheidende Erkenntnis der Studierenden aus einer Lerneinheit im Lehrwerk Lagune war nicht die gegensätzliche Einstellung zu Eulen, sondern dass Aberglaube in Deutschland im Alltag (noch) präsent ist. Das ist für die StudentInnen zunächst unverständlich; sie haben dennoch wahrgenommen, dass Aberglaube nicht nur in ihrer Kultur gegenwärtig ist, sondern auch in einem Land, das sie mit Technik, Materialismus, Pragmatismus und Fortschritt (auch mental) in Zusammenhang bringen. Sie haben Gemeinsamkeiten im gesellschaftlichen und geistigen Leben entdeckt. Hier spielt es auch keine Rolle mehr, mit welchem Ernst man dem Aberglauben in beiden Kulturräumen entgegenkommt oder welche Glaubwürdigkeit man ihm schenkt. Es entsteht eine - gewagt ausgesprochen - volkstümliche Nähe, die zunächst einmal im Fremden etwas Eigenes erkennt und das Eigene nicht mehr als etwas Rückständiges abwertet. Der Gewinn aus einer derartigen Erkenntnis: 
Gleichwertigkeit der Kulturen, Selbstachtung und ein Hinterfragen der verfestigten Bilder über die fremde (und eigene) Kultur.

- Mit dem Kennenlernen der bunten Parteienwelt im politischen System Deutschlands ist den Studierenden eine Wissenslücke gefüllt worden. Wobei der Ton auf ,bunt ${ }^{6}$ liegt. Parteien und ihre Ideologien mit Farben zu bezeichnen, ist eine andere Sicht Politik und Land zu beschreiben und zeigt ihnen auch das vielseitige parteipolitische Angebot. Die Studierenden nehmen die (entspannte) sprachliche Handhabung von relevanten und ernsthaften Themen wahr und gewinnen einen Einblick in die politische Symbolik der Farben, die nicht nur den Sprachgebrauch beeinflusst, sondern auf die Vieldeutigkeit der Sprache aufmerksam macht.

- In einem Text über den Einfluss von Fußball fällt der Name des Vereins Schalke 04. Sowohl die StudentInnen als auch die anwesenden Assistenten waren sich nicht darüber einig, ob es sich hier nur um einen fiktiven Verein handelt oder es tatsächlich einen derartigen Verein gibt. Bekannt sind unter den meisten ägyptischen Fußballfans Bayern München, Dortmund, Bayer Leverkusen. Die neue Erkenntnis, dass Schalke 04 zu den bekanntesten Vereinen der deutschen Bundesliga gehört und dass sein größter Fan Deutschlands ehemaliger Bundeskanzler Gerhard Schröder ist, war ernüchternd. Gemeint ist damit, dass berühmte Namen oder Bezeichnungen nicht gleich typisch für eine Nation oder Kultur stehen müssen, oder dass prominente Persönlichkeiten wie die eines Bundeskanzlers nicht automatisch jene berühmten Vereine favorisieren. Vorgefertigte Meinungen, Stereotype und Klischees können dadurch beseitigt werden.

Der Bruchteil an landeskundlichen Informationen oder Kulturgut, den derartiger Unterricht liefern kann, ist wichtig für den fremdsprachlichen Literaturunterricht. Es werden interkulturelle Kompetenzen erworben, die für das Verstehen von literarischen Texten notwendig sind. Die Studierenden entwickeln für das Dargestellte ein Verständnis, zumindest werden sie auf das Fremde eingestellt. Es liegt aber auch im Wesen literarischer Texte, dass sie den Lesenden auf ihre Seite gewinnen und ihre Sympathie lenken wollen. Damit ist der studierende Rezipient in der Lage, eine andere Perspektive einzunehmen, die ihm dazu verhilft, Toleranz zu entwickeln.

\section{Die Literaturvermittlung an ägyptischen Universitäten}

Der Vorgang der Vermittlung von literaturwissenschaftlichen Inhalten ist an der Universität in der Regel konventionell. Gemeint ist damit die frontale Unterweisung von wissenschaftlichen Informationen. Allerdings steht seit längerem auch die produktive Arbeit, wie sie bspw. von Ehlers (1989), Shalaby (2003/2003), Wangerin (2007), Esselborn (2010), Ziko (2012 und 2013), Bernstein/Lechner 
(2014), Augart (2015), neuerdings auch Dobstadt/Riedner (2017) u.v.a. in ihren Aufsätzen, Nachschlagewerken und Materialbänden vorgestellt werden, im Fokus der didaktischen Lehrkonzepte: durch das zeichnerische Visualisieren der räumlichen und figurellen Komponente innerhalb eines literarischen Textes, durch kreative Textarbeit, durch szenische Darstellungen analog zu Szenen aus behandelten Werken, durch die Übertragung in die Muttersprache und durch eine Vorführung. Wie Literatur sprachlich und ästhetisch funktioniert und welche Instrumente angewandt werden, wird mittels dieser induktiven Verfahren den Studierenden vermittelt, von ihnen wahrgenommen und behandelt. Dabei gilt es mitunter für den Unterricht von Literaturwissenschaft, nicht nur sprachproduktive Erträge zu gewinnen, sondern auch die literaturwissenschaftlichen Begriffe und ihren kompetenten Gebrauch in einem angemessenen literaturwissenschaftlichen Kontext zu festigen.

Die Begegnung mit literarischen Texten bzw. die literarische Erfahrung ist für den Lernprozess von entscheidender Bedeutung. Literarische Texte skizzieren und veranschaulichen - wenn auch fiktiv - Gesellschaft, Verhältnisse, zwischenmenschliche Beziehungen und alltägliche Begebenheiten der Zielkultur. Literarische Texte tragen dadurch dazu bei, die andere Kultur besser zu verstehen, ihre Anderartigkeit zu akzeptieren und auch Gemeinsamkeiten darin zu erkennen. Aber das ist ja eine längst erwiesene Weisheit. Literatur transportiert, anders ausgedrückt, das Allmenschliche, das die unterschiedlichen Kulturen gemein haben, zeigt aber auch das Typische, das der dargestellten Kultur eigen ist. An dieser Schnittstelle setzen auch die Verständigungsprobleme an und es kommt zu Fragen oder zu völlig unerwarteten Deutungen. Das macht den Literaturunterricht - für mich gesprochen - höchst interessant.

Um diese Gedanken klarzustellen, werden einige gewählte Beispiele aus fortgeschrittenen literaturwissenschaftlichen Lehrveranstaltungen vorgeführt, wo bewusst wird, wie sehr Literatur und Landeskunde im DaF-Unterricht zusammenhängen, wie sehr die Studenten darauf bedacht sind, den literarischen Text als Reflektor seiner Kultur zu verstehen, wie sehr ein literarischer Text ernst genommen und nicht als fiktiv geachtet wird und wie sehr die eigene (also fremde) Perspektive auf das Verständnis des Textes Einfluss hat und ihm neue Deutungen und Dimensionen verleiht.

\section{- Wolfgang Borchert: Die Küchenuhr}

Die Frage „Was hat eine Uhr mit einer Küche zu tun?“ wurde von einer Studentin gestellt, nicht weil das Phänomen einer ,Küchenuhr' dem Textverstehen im Wege stand. Die Tatsache der Zugehörigkeit einer Uhr in einer Küche war nicht eindeutig. Hier widersprachen ihr einige KommilitonInnen, das sei nichts Ungewöhnliches, eine Uhr in der Küche vorzufinden. Die Problematik liegt allerdings nicht darin, inwieweit diese dahin gehört, sondern darin, welchen Stellenwert der Raum „Küche' für die beschriebenen Familienverhältnisse in Borcherts Kurzgeschichte 
hat und folglich wofür die Küchenuhr im Text steht. Hier musste auf Wohnen und Wohneinrichtung eingegangen werden: z. B. wie eine Küche sein muss, worin Mahlzeiten nicht nur gekocht, sondern verzehrt werden. Sie muss, folgerten die StudentInnen, auf jeden Fall geräumig und möglichst gemütlich sein. Aber dass sich das „Leben“ sozusagen in der Küche abspielt, war auch für sie nicht selbstverständlich. Der Zusammenhang zwischen der Küchenuhr und dem Leben vor dem Einschlag der Bombe, das „Paradies“, wurde durch das Erklären (oder Aufklären) der Wohnsituation eindeutig und verständlich. Und damit stieg die Sympathie der Studierenden für die Figur und ihre Geschichte, wuchs das Verständnis für ihr Leid und wurde ihr Trümmerdasein wahrgenommen repräsentativ für die deutsche Gesellschaft in der Nachkriegszeit.

\section{- Erich Kästner: Emil und die Detektive}

Auf der Jagd nach dem Dieb wenden die Kinder zusammen mit Emil eine Reihe von Verfolgungstechniken an, die sie in der Regel beim Versteckspiel oder auch bei Räuber und Gendarme benutzen. Auf der Suche nach Analysen zu dem Roman im Internet stießen die StudentInnen auf die Erläuterung, dass Kästner hierin seine Zeit in der Armee verarbeitet hätte. Dass der Text hier eine völlig andere Reichweite gewinnt, war den StudentInnen nicht bewusst und die Interpretation wird zudem ernst genommen. Auch hier war Eingriff geboten, um ihnen eine einfachere Antwort zu geben: Kästner verarbeitete hier einfach seine Kindheit. Für den Lehrenden löste die Erläuterung aus dem Internet Schrecken, aber auch Enttäuschung aus: Erich Kästner verliert sein Profil als Pazifist - und natürlich hat das Räuber-und-Gandarme-Spiel mit etwas Militärischem zu tun, der Bezug hat sich aber schon längst entzogen: das eine steht für Krieg und Kampf, das andere für Frieden und Spiel.

Wie unnötig das Internet als Informationsquelle und glaubwürdiges Nachschlagewerk sein kann und der Vermittlung von Kultur, Literatur und Literaturwissenschaft schadet, diese gar verdirbt, ist anhand der Behandlung von Goethes Heidenröslein zu erkennen. Hier fanden die StudentInnen Interpretationen, die das Beschriebene als Gewaltakt schilderten und damit war auch das Einarbeiten gehemmt und musste wieder hergestellt werden (s. Ziko 2012).

- Heinrich Heine: Almansor

Dass Almansor seine Milchschwester heiraten will, obwohl das im Islam verboten ist, oder dass der im Exil verstorbene Vater auf dessen Wunsch nicht Richtung Mekka, sondern Richtung andalusischer Heimat begraben wurde, konnten die Studierenden nicht einsehen. Heinrich Heine hätte doch über derartige Informationen verfügen müssen, da er sich ausführlich mit dem Islam beschäftigt und über Andalusien recherchiert hatte - so ihr Argument. Dass fremdsprachliche Literatur vorrangig fiktiv ist, wird immer wieder vergessen; dass fremdsprachliche Literatur nicht nach einer Richtig-oder-Falsch-Skala beurteilt wird, schwer wahrgenommen. Die Behandlung derartiger Texte, die inhaltlich auch von den 
eigenen Wertvorstellungen und Normen abweichen, dient der (ästhetischen) Provokation. Gemeint ist hier vor allem, dass die Studierenden - im Sinn Schneiders - auch einen objektiven und sachlichen Umgang mit einem literarischen Text und seinem Inhalt lernen, um dessen Sinn zu erschließen und die vom Schriftsteller bzw. Dichter angewandten Techniken und Stoffe zu ermitteln, die er seiner Intention dienlich gemacht hat.

Literaturwissenschaftliche Erkenntnis beabsichtigt zu erklären, dass literarisches Schaffen sich nicht an die Regeln der Realität oder Norm hält. Literatur geht bewusst anders damit um, und Fiktion ist eine essentielle Beschaffenheit von literarischen Texten. Der Sinn aus einer Textwahl, die die eigenen Normen (etwas) provoziert, kann auch dazu dienen, diese Beschaffenheit den Studierenden bewusst $\mathrm{zu}$ machen. Der fremdsprachliche Lerner muss mit dem fremdsprachigen literarischen Text interagieren, um ihn auch verstehen zu können, ihm Deutungen zu verleihen und für sich neue Erkenntnisse zu erschließen, die nicht nur kultureller Natur sind, sondern auch wissenschaftlicher. Es soll die Studierenden befähigen, sich dessen Beschaffenheit zu nähern, auch wenn die beschriebenen Inhalte von den eigenen kulturellen und normativen Werten abweichen. Die Studierenden können sich so in das Wesen des literarischen Werks einfühlen und dem Text ggf. auch neue Deutungen verleihen.

\section{- Günter Grass}

Als letztes Beispiel sei ein Gedicht von Günter Grass zu erwähnen, das das Elend in einem fernöstlichen Land beschreibt. In seinem 1988 erschienenen Werk Zunge zeigen. Ein Tagebuch in Zeichnungen, Prosa und einem Gedicht wird seine Kritik gegenüber der westlichen Welt deutlich, deren luxeriöses Leben durch Unzufriedenheit gekennzeichnet ist (vgl. Baumann/Oberle 1996: 250).

Gegenwärtig jedoch

fanden wir mitten im Müll, wo er geebnet

schon Humus zu werden

und Gemüse zu treiben verspricht,

eine Schule in einem Schuppen versteckt.

Still hockten Müllkinder über Schiefertafeln

und übten bengalische Schrift.

das Leben ist schön, hieß (für uns) übersetzt,

was sie zur Übung wieder und wieder

schrieben. (aus: Baumann/Oberle 1996: 250)

Grass schildert in seinem Gedicht Kinder, die inmitten von Müllhalden lesen und schreiben lernen. Die StudentInnen wissend über die Kritik von Grass hatten ihre eigene Perspektive: Grass möchte eine Welt zeigen, die von Hoffnung geprägt ist. So wie inmitten des Mülls Pflanzen gedeihen, gedeiht auch der Verstand der jungen Schüler in ihrer Schulhütte. Diese positiv wertende Interpretation der Studierenden steht in Opposition zu der negativen Haltung Grass ' seiner Gesellschaft gegenüber. Die Auslegung der StudentInnen ist auf ihre Erfahrung zurückzuführen, die sich mit 
ärmlichen Bedingungen auskennt. Die zielsprachliche Perspektive hat demnach nicht die eigene Einstellung zum Gedicht beeinflusst, vielmehr die Studierenden ermutigt, ihm eine andere Sichtweise zu verleihen und eine andere Interpretation zu ergänzen.

\section{Erkenntnisse und Konsequenzen für den akademischen Literaturunterricht}

Welche Erkenntnisse und Konsequenzen aus der langjährigen Erfahrung mit Literatur und Literaturwissenschaft im universitären Kontext erschlossen wurden, sollen im Folgenden als Anregung für weitere Diskussion verstanden werden.

Die Studierenden sind am literarischen Inhalt interessiert und auf das Vermittelte neugierig, weil es Unerwartetes, Ungewohntes mit sich bringt, weil es sie in die Welt der Zielkultur hineinversetzt und weil damit auch ästhetische Werte verbunden sind. Die Studenten haben ein Verlangen entwickelt, den Grund dieser Eigenart der fremden Welt, des Zielsprachenlandes $\mathrm{zu}$ verstehen. Die Fremdwahrnehmung des Fremdsprachenlerners kann zwar zur literarischen Intention des Schriftstellers führen. Fremdwahrnehmung ist aber andererseits auch ein Weg der anderen Deutung, die oft auf die eigene Erfahrung beruht und aus der eigenen Perspektive entspringt. Sie erlaubt Deutungen, die durchaus bereichernd sind.

Das heißt für die Lehrkraft weiter: Selbstverständlichkeiten gibt es nicht im fremdsprachlichen Literaturunterricht; es ist aber auch gesund für den Lehrenden (wie auch für die wissenschaftliche Beschäftigung damit) davon auszugehen. Denn nur so realisiert man, was fremd für die Lernenden ist. Es ist ein fortwährender Prozess, im fremdsprachlichen Literaturunterricht landeskundliche und kuturelle, ästhetische und literaturwissenschaftliche Lücken zu füllen. Damit werden neben den vorab festgesetzten Zielen jeder literaturwissenschaftlichen Veranstaltungen neue hinzugefügt und in die nächsten eingebunden.

Die zeitliche Positionierung des literarischen Textes spielt im fremdsprachlichen Literaturunterricht eine wichtige Rolle, das Geschilderte $\mathrm{zu}$ verstehen. Nicht umsonst hat man sich in zahlreichen Beiträgen mit dem literarischen Kanon und der Auswahl von bestimmten literarischen Werken beschäftigt, worin insbesondere die Gegenwartsliteratur und die Migrationsliteratur favorisiert werden (dazu bspw. Cerri 2007; Rösch 2011 u.v.m.). Die gängige Überzeugung besagt, dass literarische Werke aus der Gegenwartsliteratur für Studierende verständlicher und ihren Inhalten her nachvollziehbarer sind als ältere Texte. Allerdings gerät dieser Faktor in den Hintergrund, wenn ältere Texte die Gegenwart der Studierenden ansprechen: z. B. Büchners Dantons Tod während der Januar-Revolution in Ägypten (s. Ziko 2013), Heines Almansor (in Bezug auf Diskriminierung, Fremdheit, Hass) wie auch Brechts Galelei (in Bezug auf Wahrheit) werfen ihre Schatten auf die Gegenwart der Studierenden. 
Im Hinblick auf die Informationsquellen für literarische Werke ist didaktisch betrachtet das Internet ein Hindernis für einen geplanten Unterrichtsverlauf, der literaturwissenschaftliche Inhalte vermitteln möchte. Denn während der Lehrende auf Strategien setzt, die die Studierenden das Verstehen des literarischen Textes einleiten und die sie zu einem wissenschaftlichen Umgang mit Literatur befähigen sollen, klammern sich Lernende an die Internet-Interpretationen fest. Zum einen weil sie mit einem guten Deutsch die Prüfung bestehen wollen, zum anderen weil sie sich die Mühe des eigenständigen Arbeitens ersparen möchten. Selbstinitiativen, kreativer Umgang und produktives Arbeiten bleiben dabei auf der Strecke.

Die Schlussfrage, die sich hier nach der Verbindung von Literatur- und Kulturvermittlung und Berufsperspektive und Berufsausübung stellt, bringt uns zu den Zielen des Deutsch-Studiums an den ägyptischen Universitäten zurück.

Der Absolvent ist auch als Vermittler der deutschsprachigen Kultur auszubilden. Nach dem Studium hat er die Möglichkeit, in den Lehrerberuf einzusteigen, sich in der Tourismusbranche zu verwirklichen oder als Übersetzer durchzusetzen. Das sind die klassischen Berufsperspektiven. Neuerdings spielen insbesondere Firmen, die einen deutschsprachigen Telefonkundendienst anbieten, eine vielversprechende und lukrative Rolle für die Absolventen. Andere Absolventen wiederum finden Jobs fernab von der deutschen Sprache.

Auf die Frage, ob Literatur den StudentInnen nach dem Studium nützlich sein kann, meinen viele aus dem 4. Studienjahr: Nur wenn man Postgraduale Studien machen oder als Lehrer arbeiten möchte. Andere allerdings sind zur Erkenntnis gekommen, dass der literaturwissenschaftliche Unterricht ihnen Fertigkeiten vermittelt, die oft mit dem Verstehen von anderen Mitmenschen verbunden sind.

Mit den erworbenen Analysetechniken meinen einige Studenten und Absolventen ferner, dass sie sich Strategien auch für den Alltag angeeignet haben: Sie lernen Menschen besser verstehen oder erwarten gar, wie sie reagieren. Das Erlernen und Anwenden von wissenschaftlichem Arbeiten und Denken sowie die Unterscheidung zwischen spontanem Handeln und logisch-systematischem Arbeiten zählen zu den wichtigsten Zielen eines Studiums, denn sie sollten über das Studium hinaus reichen, also die angehenden Absolventen für ihre berufliche Entwicklung vorbereiten.

Der Erwerb von fachwissenschaftlichen Inhalten der Literatur lehrt die Studierenden nicht nur den Text zu verstehen, der Text gewinnt mittels seiner literarischen Wesensmerkmale weitere Bedeutungen und verhilft dazu, den Erkenntnishorizont der Studierenden zu erweitern. Sie lernen zudem, dass Texte jeglicher Art Werkzeuge benötigen, um ihre Aussage weiterzuleiten und hervorzuheben; und dass sie Techniken brauchen, um diese Aussage zu verstehen, $\mathrm{zu}$ analysieren und dazu Stellung $\mathrm{zu}$ nehmen. Noch wichtiger ist, dass der Studierende den literarischen Text zunächst als eigene Welt zu respektieren lernt, in dem auch manchmal etwas über die Außenwelt, in der er entstanden ist, gesagt 
wird. Der Text ist zwar fiktiv, er ist jedoch aus einer realen Welt entstanden (gesellschaftlichen, landschaftlichen, politischen, religiösen, historischen u.a. Verhältnissen und Umgebungen).

Konsequenterweise heißt das, im Literaturunterricht das form- und normbezogene Verfahren nicht aufzugeben - im Sinne eines wissenschaftlichen Einführens in die Techniken des literarischen Analysierens, wie z. B. der Figurenanalyse, der Inhaltsanalyse, der Analyse lyrischer, prosaischer und dramatischer Text, deren Merkmale und Stile vorzuführen und zu veranschaulichen. Methodenvielfalt muss im Rahmen eines literaturdidaktischen Vorhabens konzeptionell zum Einsatz kommen und schließlich sind Konsequenzen aus jedem einzelnen Vorhaben zu ziehen, von denen Lernende und Lehrende Gebrauch machen können, die bei produktivem Arbeiten und der Verfeinerung von Skills, bei der Ausweitung der Perspektive und der Sensibilisierung für den ästhetischen Gehalt eingesetzt werden können.

Das alles ist durchaus idealistisch, gehört aber auch zum „Tun“ jeder akademischen Praxis. Ich möchte erneut daran erinnern, dass die Arbeit an den Universitäten vorrangig auch die berufliche Ausbildung einleitet. Wissenschaftliches Arbeiten ist damit auch eine Voraussetzung für die spätere Berufspraxis. Produktive Tätigkeit und kreativer Umgang an und mit literarischen Texten dienen der Lösungsfindung und der intensiven und annäherenden Auseinandersetzung mit Problemfeldern und der Konfliktbewältigung. Interkulturelle Kompetenzen wie Perpektivenwechsel, Toleranz, Empathie, Identitätsdarstellung u.ä. sind durchaus auch wichtig für den Umgang in der Arbeitswelt. Aber am Ende bleibt es doch am Arbeitsmarkt und am Charakter des Absolventen, was aus dem Gelernten und Erworbenen gemacht wird. Was dann von der Vorstellung über Kultur und Bild der gelernten Sprache Deutsch geblieben ist, hängt von der Beschäftigung des Absolventen ab. Die Ziele der eigenen akademischen Leistungen als Lehrkraft sind also nicht immer ganz einkalkulierbar, das gilt allerdings auch für jedes didaktische Tun.

\section{Fazit}

Aus der vorangegangenen Darstellung der Situation des Studiums der deutschen Sprache und Literatur an ägyptischen Universitäten und erschlossenen Konsequenzen für Lehre und Forschung in demselben ist folgendes zu rekapitulieren:

- Die Rahmenbedingungen für Literatur- und Kulturvermittlung innerhalb des Faches DaF bzw. Interkulturelle Germanistik u.ä. Bezeichnungen, die die Germanistik außerhalb des deutschsprachigen Raumes meinen und beschreiben, sind hinsichtlich der Erfordernisse der sprachlichen Kompetenz der Studierenden, Inhalte und Ziele des Literaturunterrichts, 
Forschungsschwerpunkte, Methodenauswahl und schließlich Erwartungen der Studierenden und Erfordernisse des Arbeitsmarkts tiefer zu ergründen.

- Es genügt für die Literaturwissenschaft eines Faches DaF allein nicht aus, didaktische Modelle vorzustellen oder interpretatorische Fremdperspektiven zu präsentieren. Eine theoretische Auseinandersetzung nimmt zum einen den Tatbestand wahr und fördert eine fundierte Weiterentwicklung des Faches auch außerhalb des deutschsprachigen Raumes.

- Die Frage nach der Möglichkeit, die sprachliche Barriere bei der Arbeit mit literarischen Texten zu beheben, ist damit verbunden, eine Vermittlung kulturwissenschaftlicher, soziohistorischer und auch landeskundlicher Inhalte zu ermöglichen. Diese sollten allerdings bereits im Grundstudium eintreten und nicht auf ein postgraduales Studium verschoben werden. Dabei müssten evtl. für die ägyptische Germanistik bahnbrechende oder gar revolutionierende didaktische Lösungen gefunden werden.

- In diesem Zusammenhang ist das Verständnis von Literaturwissenschaft als Kulturwissenschaft ein grundlegendes für die Lehrkraft, nicht aber unbedingt für den Studierenden.

- Die Kenntnis über Literaturwissenschaft ist ein notwendiges Instrumentarium nicht allein für den Literatur(wissenschafts)betrieb, sondern auch für eine professionelle berufsorientierte Ausbildung.

- Eine wichtige Erkenntnis für die Studierenden der deutschen Sprache und Literatur: Die Fiktivität eines fremdsprachigen literarischen Textes unabhängig von seiner zeitlichen Entstehung - schließt seine kulturrepräsentative Funktion mit ein. Diese Repräsentation realisiert sich allerdings nicht allein kulturell, sondern steht im Schatten mentalgeschichtlicher, textproduktiver und/oder autorintentioneller Bedingungen während seiner Entstehung. Die kulturelle Repräsentativität eines literarischen Textes ist demnach relativ.

Die universitäre fremdsprachliche Literaturdidaktik entpuppt sich in diesem Sinne als eine wahre Herausforderung. Um ihr Herr zu werden, sind neue Einsichten zu gewinnen, die bislang in der Germanistik- und DaF-Diskussion im deutschsprachigen Geographieraum besprochen werden. Die ägyptische Germanistik ist in jüngster Zeit darauf gestoßen und benötigt eine Fortsetzung der Diskussion in eigener Sache. Dieser Gedanke steht im Zusammenhang dazu, wie man zum Fach DaF steht und wie man ihn versteht: als bloße Fremdsprachenvermitllung oder als eine fremdsprachenphilologische Auseinandersetzung. Auch wenn beide Sichtweisen beim Prozess des Spracherwerbs Literatur, Kultur und berufliche Komptenzen einbinden (wollen), so gehört die erste in ein Sprachinstitut höchstens in eine Schule, aber nicht wie die zweite in eine akademische Einrichtung. 


\section{Literatur in Auswahl}

Ali, Adel 2009: Bildergeschichten im DaF-Unterricht. Förderung sprachlicher Kompetenz und Kreativität. In: Info DaF 36/5, 462-478.

Altmayer, Claus 1997: Gibt es eine Literaturwissenschaft des Faches Deutsch als Fremdsprache? Ein Beitrag zur Strukturdebatte. In: Deutsch als Fremdsprache 34/4, 198-203.

Altmayer, Claus 2002: Kulturelle Deutungsmuster in Texten. Prinzipien und Verfahren einer kulturwissenschaftlichen Textanalyse im Fach Deutsch als Fremdsprache. In: Zeitschrift für Interkulturellen Fremdsprachenunterricht [Online], 6(3), 25 pp. Available: http://www.spz.tudarmstadt.de/projekt_ejournal/jg_06_3/beitrag/deutungsmuster.htm [Stand: 03.08.2017].

Altmayer, Claus; Dobstadt, Michael; Riedner, Renate; Schier, Carmen 2014 (Hrsg.): Literatur in Deutsch als Fremdsprache und internationaler Germanistik. Konzepte, Themen, Forschungsperspektiven. Tübingen: Stauffenburg Verlag.

Altmayer, Claus; Dobstadt, Michael; Riedner; Renate 2014: Literatur in sprachund kulturbezogenen Lehr- und Lernprozessen im Kontext von DaF/DaZ. Eine Einführung in den Themenschwerpunkt. In: Deutsch als Fremdsprache 51/1, 310.

Aufderstraße, Hartmut; Müller, Jutta; Storz, Thomas 2008: Lagune 1. Kursbuch. Ismaningen: Hueber Verlag.

Augart, Julia 2015: Literaturvermittlung - kreativ. Beispiele zum Umgang mit Literatur im Südlichen Afrika. In: Info DaF, 42/6, 576-590.

Baumann, Barbara; Oberle, Brigitta 1996: Deutsche Literatur in Epochen. 2. überarbeitete Auflage. Ismaning: Max Hueber Verlag.

Bernstein, Nils; Lerchner, Charlotte 2014 (Hrsg.): Ästhetisches Lernen. im DaF/DaZ-Unterricht. Literatur - Theater - Bildende Kunst - Musik - Film. Materialien Deutsch als Fremdsprache. Band 93. Göttingen: Universitätsverlag Göttingen.

Bredella, Lothar 2002: Literarisches und interkulturelles Verstehen. Tübingen.

Bredella, Lothar 2007: Fremde Welten entwerfen. Der Bildungssinn literarischer Texte. In: Fäcke/Wangerin (Hrsg.), 30-50.

Cerri, Chiara 2011: Mut zur interkulturellen Literatur im DaF-Unterricht. In: Info DaF 38/4, 391-413.

Choi, Young-Jin 2002: Literatur im universitären DaF-Unterricht in Korea. Zur Verzahnung von Theorie und Praxis. In: Info DaF 29/6, 556-566.

Czucka, Eckehard 1993: Schrift-Kultur und fremde Sprache. Überlegungen zum Literaturunterricht mit ägyptischen Deutschstudenten. In: Kairoer Germanistische Studien 7, 69-84. 
Czucka, Eckehard; Neuland, Eva 2011 (Hrsg.): Interkulturelle Kommunikation. Perspektiven einer anwendungsorientierten Germanistik. München: iudicium Verlag GmbH.

Ehlers, Swantje 1989: Sehen lernen. Zur ästhetischen Erfahrung im Kontext interkultureller Literaturvermittlung. In: Jahrbuch Deutsch als Fremdsprache 14, 171-197.

Ehlers, Swantje 2010: Literarische Texte im Deutsch als Fremd- und Zweitsprache-Unterricht: Gegenstände und Ansätze. In: Krumm/Fandrych/Hufeisen/Riemer (Hrsg.), 1530-1544.

El Dib, Nahed 2011: Lachende Pessimisten aus Osten und Westen. Probleme des interkulturellen Dialogs und Möglichkeiten einer interkulturellen Kommunikation. In: Czucka/Neuland (Hrsg.), 180-193.

Esselborn, Karl 2010: Interkulturelle Literaturvermittlung zwischen didaktischer Theorie und Praxis. München: IUDICIUM Verlag GmbH.

Ewert, Michael 2010: Literarischer Kanon und Fragen der Literaturvermittlung. In: Krumm/Fandrych/Hufeisen/Riemer (Hrsg.),1555-1565.

Fäcke, Christiane; Wangerin, Wolfgang 2007 (Hrsg.): Neue Wege zu und mit literarischen Texten. (Diskussionsforum Deutsch 25). Baltmannsweiler: Schneider Hohengehren.

Hofmann, Michael 2006: Interkulturelle Literaturwissenschaft. Eine Einführung. Paderborn: Wilhelm Fink Verlag.

Helal, Salah 2011: Das Bild der Araber und Muslime im Werk Elias Canettis aus der Sicht eines arabischen Germanisten. In: Czucka/Neuland (Hrsg.), 194-207.

Khattab, Aleya 2010: Deutsch in Ägypten. In: Krumm/Fandrych/Hufeisen/Riemer (Hrsg.), 1602-1606.

Kramsch, Claire 2011: Symbolische Kompetenz durch literarische Texte. In: Fremdsprache Deutsch 44, 35-40.

Kramsch, Claire 2016: Symbolische Kompetenz im Zeitalter der Mehrsprachigkeit. Abrufbar unter: bielefeld.de/lili/studium/faecher/daf/gastlehrstuhl-harald-

weinrich/Claire_Kramsch_Rede_Harald-Weinrich-Gastlehrstuhl.pdf, $8 \mathrm{pp}$. [Stand:18.4.2018].

Krumm, Hans-Jürgen; Fandrych, Christian; Hufeisen, Britta; Riemer, Claudia 2010 (Hrsg.): Deutsch als Fremd- und Zweitsprache. Ein internationales Handbuch. Band 2.

Maher, Moustafa 2007: Die ägyptische Germanistik zwischen historischem Momentum und Suche nach neuen Perspektiven. In: KGS 17, Kongressakten des 2. Internationalen Germanistik-Kongresses „Wege über Grenzen. Perspektiven der Germanistik“, 2.-4. April 2007, Kairo. Teil I, 3-17.

Massoud, Fatma 2011: Interkulturelle Kommunikation in Ägypten. One way ticket oder ,hin und zurück'? In: Czucka/Neuland (Hrsg.), 147-160. 
Riedner, Renate 2010: Literatur, Kultur, Leser und Fremde - Theoriebildung und Literaturvermittlung im Fach Deutsch als Fremd- und Zweitsprache. In: Krumm/Fandrych/Hufeisen/Riemer (Hrsg.), 1544-1554.

Rösch, Heidi 2007: Interkulturelle Literatur lesen. Literatur interkulturell lesen. In: Fäcke/Wangerin (Hrsg.), 51-62.

Rösch, Heidi 2016: Glokale Literatur im (Deutsch-als-) Fremdsprachunterricht. In: Info DaF 43/1, 3-20.

Salama, Dalia Aboul Fotouh 2011: Postkolonialistische Perspektiven in der deutschen und arabischen Literatur. Zu Uwe Timms „Morenga“ und Baha Tahers „Oase des Sonnenuntergangs“. In: Czucka/Neuland (Hrsg.), 208-224.

Shalaby, Iman 2002/2003: Ästhetisch-kreative Entfaltung von Sinn. Zum Stellenwert der Konkreten Poesie bei der Sensibilisierung ägyptischer Deutschlehrer-Studenten für deutschsprachliche Bedeutungsdifferenzierung. In: Kairoer Germanistische Studien 13, 247-296.

Schiedermair, Simone 2011: Literarische Texte als literarische Texte. Vieldeutigkeit, Anschaulichkeit, Kontextverbundenheit. In: Fremdsprache Deutsch 44, 28-34.

Schneider, Jost: Einführung in die moderne Literaturwissenschaft. 2. durchgelesene Auflage. Bielefeld: Aisthesis Verlag.

Spinner, Kasper H. 2007: Lesestrategien oder literarisches Gespräch? In: Fäcke/Wangerin (Hrsg.),18-29.

Tütken, Gisela 2006: Literatur im Unterricht Deutsch als Fremdsprache an der Hochschule im Ausland - aber wie? Ein Vorschlag am Beispiel Russlands: In: Info DaF 33/1, 52-90.

Wangerin, Wolfgang 2007: Der Leser ,,macht eigentlich aus einem Buche, was er will“ (Novalis). Konstruktivistische Begründungen produktiven Literaturunterrichts. In: Fäcke/Wangerin (Hrsg.), 63-88.

Wierlacher, Alois 1985: Das Fremde und das Eigene. Prolegomena zu einer interkulturellen Germanistik. München: iudicium.

Ziko, Lamyaa Abdelmohsen O. A. 2012: Lyrik im universitären DaF-Unterricht in Ägypten. In: Faculty of Arts Journal, Edition No. 90, July 2012, AlMenoufia University, 3-24.

Ziko, Lamyaa Abdelmohsen O. A. 2013: Dantons Tod. Lektüre im literarischen DaF-Unterricht im Rahmen der 25. Januar-Revolution von Ägypten. In: Zielsprache Deutsch, 40/3, 19-33. 